Results: In total, 32 patients were included: endometrioid $(n=18)$, serous $(n=5)$, carcinosarcoma $(n=6)$, and others $(n=3)$. Among them, 25 (78.1\%) had stage IVB disease. The most common NAC regimen was paclitaxel-carboplatin $(n=25$, $78.1 \%$ ), administered with median of 6 cycles. While 26 $(81.3 \%)$ showed an objective response, 2 (6.3\%) progressed despite NAC. At the time of IDS, 23 (71.9\%) achieved complete cytoreduction. During 30.5 months of the median followup, there were 23 recurrences and 7 deaths, corresponding to 19 months of median PFS and $46.7 \%$ of 3 -year OS rate. In multivariate analysis, endometrioid histology was associated with better PFS (hazard ratio $[H R]=0.181 ; \mathrm{p}=0.001$ ) and OS (HR=0.112; $\mathrm{p}=0.029$ ), while complete cytoreduction significantly improved PFS (HR=0.258; $\mathrm{p}=0.008)$. Conclusion: We found NAC-IDS is feasible and effective in unresectable, metastatic endometrial cancer. Tumor histology and the possibility of complete cytoreduction would be the primary considerations for NAC-IDS.

Oral (OM4)

Endometrial Hyperplasia, Endometrial Intra-epithelial Neoplasia, and Endometrial Cancer

https://doi.org/10.3802/jgo.2021.32.S1.0M4

\section{Comparison of sentinel lymph node biopsy alone to complete lymphadenectomy in endometrial cancer patients: a retrospective study}

\section{Amany Abdelwadoud Makroum, ${ }^{1, *}$ Jung-Yun Lee, ${ }^{2}$ Eun Ji Nam, ${ }^{2}$ Sunghoon Kim, ${ }^{2}$ Sang Wun Kim, ${ }^{2}$ Young Tae Kim ${ }^{2}$ \\ 'Department of Obstetrics and Gynecology, Faculty of Medicine, Mansoura University, Mansoura, Egypt (amany_makroum@mans.edu.eg) ${ }^{2}$ Department of Obstetrics and Gynecology, Institute of Women's Life Medical Science, Yonsei University College of Medicine, Seoul, Korea}

Objective: Sentinel lymph node (SLN) biopsy allows to spare node negative patients from surgical comorbidities. Our objectives were evaluation of outcomes of SLN biopsy only compared to additional lymph node (LN) dissection in endometrial cancer patients.

Methods: Single institutional retrospective study of endometroid type of endometrial carcinoma patients at Yonsei Cancer Center who underwent minimally invasive surgical staging with SLN biopsy with or without additional LN dissection between January 2015 to December 2019.

Results: A total of 301 patients were included in this study. Eighty-two underwent SLN biopsy only and were compared to 219 with extra LN dissection. The median age was 53 years and median body mass index was $25 \mathrm{~kg} / \mathrm{m}^{2}$. In terms of operative outcomes, SLN biopsy only group showed statistically significant decreased surgical time $(\mathrm{p}=0.001)$. However, estimated blood loss during operation did not show any statistically significant difference. The sites and numbers of SLN did not show statistically significant difference. There was one case with LN metastasis from whom SLN metastasis was not detected. This case reflects false negativity of SLN biopsy and overall false negative rate of SLN biopsy was $0.45 \%$. Recurrence and death events did not show any statistically significant difference between the two groups during the follow-up period. Conclusion: This study showed similar prognosis among the 2 included groups with acceptable diagnostic accuracy of SLN biopsy with small detected false negative rates. Thus, LN assessment can be considered as valuable and could safely replace $\mathrm{LN}$ dissection.

Oral (OM5)

Endometrial Hyperplasia, Endometrial Intra-epithelial Neoplasia, and Endometrial Cancer

https://doi.org/10.3802/jgo.2021.32.S1.0M5

\section{The fluorescence imaging for laparoscopic and laparotomic endometrial sentinel lymph node biopsy (FILLES) trial: Siriraj Gynecologic sentinel Node of endometrial cancer (SiGN-En) study}

\section{Khemanat Khemworapong, "Atthapon Jaishuen, Mongkol Benjapibal, Perapong Inthasorn, Boonlert Viriyapak, Pisutt Srichaikul, Nida Jareemit, Malee Warnnissorn, Suchanan Hanamornroongruang \\ Mahidol University, Nakhon Pathom, Thailand (khemanatkhem@gmail.com)}

Objective: To establish the detection rate of sentinel lymph node (SLN) biopsies and to determine the sensitivity and false-negative rate of SLN biopsies compared with those of systematic pelvic and para-aortic lymphadenectomies in endometrial cancer.

Methods: This prospective cohort study enrolled patients with endometrial cancer who were scheduled for surgical staging. Patients with a history of chemotherapy or radiotherapy, an abnormal liver function test, or an allergy to indocyanine green (ICG) were excluded. All patients underwent surgical staging with an ICG injection at the cervix. SLNs were identified by a near-infrared fluorescent camera. All SLNs were sent to a pathologist for ultra-staging.

Results: From November 2019 to March 2021, 101 patients underwent SLN mapping and surgical staging. SLNs were not detected bilaterally in 2 patients. The detection rate of the SLN biopsies in this study was $97.9 \%$. Thus, the accuracy of the SLN biopsies was $98.9 \%$. The sensitivity for finding metastatic SLNs 
was $92.3 \%$, with a negative predictive value of $98.8 \%$. Conclusion: A SLN biopsy in endometrial cancer has a high detection rate and high accuracy. However, surgical expertise and a learning curve are required.

Oral (OM6)

Uterine Sarcoma

https://doi.org/10.3802/jgo.2021.32.S1.0M6

\section{Impact of power morcellation and tissue extraction technique in women with unexpected uterine cancer after uterine- conserving surgery}

Hyun-Woong Cho, Jae Kwan Lee, Jin Hwa Hong*

Korea University Guro Hospital, Seoul, Korea (jhhong93@korea.ac.kr)

Objective: Although there is a concern about laparoscopic uterus-conserving surgery (UCS) including power morcellation which may adversely affect outcome in patients with unexpected uterine cancer, the impact of power morcellation and tissue extraction technique on prognosis is still indeterminate based on existing evidence. The aim of this study is to evaluate the impact of type of surgery on prognosis with interest in the change of morcellation technique.

Methods: Using Korea National Health Insurance (KNHI) database, we identified 1,788 women with unexpected uterine cancer who underwent a hysterectomy or UCS for presumed benign disease from January 2003 to December 2018. The comparison of mortality between hysterectomy and UCS was assessed according to laparoscopic and open surgery. Cox proportional hazards models were used to estimate the adjusted hazard ratios (aHRs) and $95 \%$ confidence intervals (CIs) for the mortality of patients. Results: Laparoscopic UCS was associated with a higher risk for mortality than laparoscopic hysterectomy ( $\mathrm{aHR}=3.03$; 95\% $\mathrm{CI}=1.25-7.34)$, whereas there was no significant association between open hysterectomy and UCS. The survival of laparoscopic UCS after Food and Drug Administration warning in 2014 was higher than that of laparoscopic UCS before 2014 (4-year survival rate: $100 \%$ [7/7] vs. $77.4 \%$ [24/31]). There was no significant difference of survival rate between open UCS before and after 2014.

Conclusion: Uncontained power morcellation could be associated to increased risk of mortality in women with unexpected uterine cancer. Preoperative evaluation of patents is required to reduce the effect of unexpected uterine cancer. For women who incidental uterine cancer is suspected, careful surgical procedures that can prevent tumor dissemination may improve the prognosis.
Oral (OM7)

Endometrial Hyperplasia, Endometrial Intra-epithelial Neoplasia, and Endometrial Cancer

https://doi.org/10.3802/jgo.2021.32.S1.0M7

\section{Clinicopathological factors and IHC markers predicting survival outcomes of uterine endometrial carcinoma: experience of a gynaecologic oncology unit from India}

\author{
Megha Nandwani, ${ }^{1, *}$ Hemlata Garg, ${ }^{2}$ Satinder Kaur ${ }^{3}$ \\ 'Narayana Superspeciality Hospital, Gurugram, India \\ (megha.nandwani@gmail.com) \\ ${ }^{2}$ Narayana Superspeciality Hospital, Dharamshila, India \\ ${ }^{3}$ Narayana Superspeciality Hospital, New Delhi, India
}

Objective: To study the clinicopathological factors and immunohistochemistry (IHC) markers in predicting survival outcome of women with endometrial carcinoma undergoing primary surgery. Methods: All cases of carcinoma endometrium diagnosed and treated at our hospital from 1st July 2013 to 30th June 2020 were studied and retrospectively reviewed for demographic profile and survival analysis. The factors influencing survival were critically evaluated. All histopathology slides were made to undergo IHC staining with p53 marker and microsatellite instability (MSI) markers (MLH1, MSH2, MSH6) and its correlation with survival was analyzed. Results: A total of 84 cases of carcinoma endometrium were evaluated. More than $50 \%$ patients belonged to age group of 60 years and above and were postmenopausal. The factors influencing survival were evaluated using multivariate analysis. These factors were age, stage, myometrial invasion and lymph node metastasis. The overall survival of our study population was $91.5 \%$. IHC markers like p53 and MLH1, MSH2, MSH6 were useful in predicting survival outcomes and prognosis in our study population.

Conclusion: Endometrial cancer have good survival outcomes when diagnosed and treated at an earlier stage. Factors influencing survival include age, myometrial invasion and lymph node metastasis. IHC markers; p53 and MSI markers like MLH1, MSH2, MSH6 are helpful in not only predicting survival outcome but also in guiding adjuvant treatment in these patients.

Oral (OM8)

Endometrial Hyperplasia, Endometrial Intra-epithelial Neoplasia, and Endometrial Cancer

https://doi.org/10.3802/jgo.2021.32.S1.0M8

\section{Retreatment with progestin for recurrence after achieving complete response with fertility sparing hormonal treatment in patients with early endometrial cancer}

